\title{
Analysis of complications associated with epidural analgesia - a 5-year audit
}

\author{
Authors: Rui Freitas Silva; Co-Authors: Hernâni Resendes, Tiago Cabral, Clara Lima, \\ João Simas, João Borges. \\ Institution: Hospital do Divino Espírito Santo, Azores, Portugal.
}

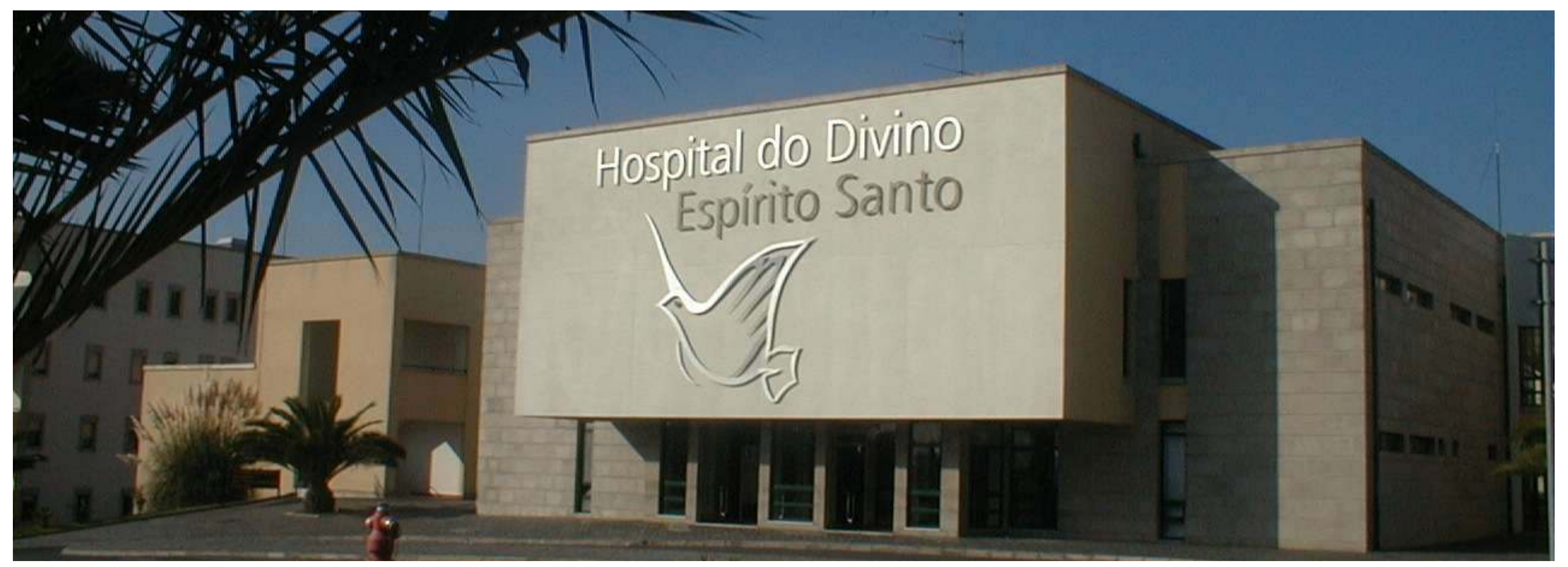

\section{Introduction:}

Epidural analgesia is a widely used method in the treatment of postoperative pain. Its increasing use is due to the high efficacy and the low rate of associated complications. The aim of this study was to evaluate the records of the Acute Pain Service (APS) of Divino Espírito Santo Hospital (DESH) over a period of 5 years, in order to determine: 1) the number of epidural catheters used for post operative analgesia; 2) the complications associated with their use.

\section{Methods:}

Retrospective study using an Excel database of APS registries from June 2011 to September 2016.

\section{Results:}

1) During the referred period the APS followed a total of 10866 patients, to which 29778 follow-up visits were performed; 4010 epidural catheters were used for intra and postoperative analgesia.

2) Trough the analysis of the records we were able to identify 189 complications associated with the catheter or drugs administered by the epidural route including: 99 accidental exteriorizations, 21 episodes of severe hypotension, 11 cases of nausea and vomiting, 10 dura punctures, 9 non-functioning catheters and 9 cases of severe pruritus (Table 1).

3) There were also 8 deaths while undergoing analgesic protocol, 5 cases of paresthesia of lower limbs, 4 urinary retentions, 2 cases of hallucinations and 2 cases of bradycardia. We also identified isolated cases (1) of: suspected allergy to the local anesthetic, intense and prolonged motor block, seizures after administration of epidural drug, paralytic ileus, lipothymia, respiratory arrest, signs of inflammatory reaction at the insertion site, suspected epidural hematoma (unconfirmed) and pulmonary thromboembolism.
Table 1: Complications associated with the epidural catheter or drugs administered by the epidural route.

\begin{tabular}{|l|l|}
\hline Complication & Number of cases \\
\hline Accidental exteriorization & 99 \\
\hline Severe hypotension & 21 \\
\hline Nausea and vomiting & 11 \\
\hline Dura punctures & 10 \\
\hline Non-functioning catheters & 9 \\
\hline Severe pruritus & 9 \\
\hline Total & 189 \\
\hline
\end{tabular}

\section{Discussion/Conclusion:}

Epidural analgesia is not free of risks and complications, so that the audits of the registries performed are important. ${ }^{1}$ From the analysis performed it was possible to draw the following conclusions:

- In DESH epidural analgesia is a widely used modality for the treatment of acute postoperative pain;

- $\quad$ From the analysis of the records, it was concluded that there was no mortality directly associated with the placement and use of epidural catheters.

- The cases of dura mater puncture, when symptomatic and resistant to non-invasive treatment, were treated with blood patch followed by resolution of symptoms.

- Incomplete or missing records are a limitation to the full assessment of the reported complications and their systematic implementation should be encouraged among all service providers. 